\title{
ON A MULTIPLICATION DECOMPOSITION THEOREM IN A DEDEKIND $\sigma$-COMPLETE PARTIALLY ORDERED LINEAR ALGEBRA
}

\author{
TAEN-YU DAI
}

\begin{abstract}
Suppose a Dedekind $\sigma$-complete partially ordered linear algebra (dsc-pola) satisfies a certain multiplication decomposition property (see definition below), then we show that this partially ordered linear algebra actually has the same structure of a special class of real matrix algebras, consisting of elements that can be decomposed as diagonal part plus nilpotent part $w$, such that $w^{2}=0$.
\end{abstract}

A dsc-pola, denoted by $A$ (or $B$ ) is a real linear associative algebra which satisfies the following two conditions: (1) It is partially ordered so that it is a directed partially ordered linear space and $0 \leqq x y$ whenever $x, y \in A$, $0 \leqq x, 0 \leqq y$. (2) It is Dedekind $\sigma$-complete, i.e., if $x_{n} \in A, 0 \leqq \cdots \leqq x_{2} \leqq x_{1}$, then $\inf \left\{x_{n}\right\}$ exists. A dsc-pola $A$ has the Archimedean property: If $x$, $y \in A$ and $n x \leqq y$ for every positive integer $n$, then $x \leqq 0$. In this paper we will assume $A$ has a multiplicative identity $1 \geqq 0$. Let $I=\{y: y \geqq 1$, and $\left.y^{-1} \geqq 0\right\} \subset A$. Define $A_{1}=\bigcup_{y \in I}\{x:-y \leqq x \leqq y\}$. Then it was shown by $\mathrm{R}$. DeMarr that the multiplication of the elements in $A_{1}$ is commutative, and $A_{1}$ behaves much like an algebra of real-valued functions; moreover, $A_{1}$ is a lattice and has no nonzero nilpotent. For the details of the proofs and examples of $A_{1}$ we refer to [2]. (Note in [2], instead of the term dsc-pola, we use polac; actually they have the same meaning.) We will call $A_{1}$ the functional or diagonal part of $A$. Let $A$ be a dsc-pola which has the following multiplication decomposition property (abbreviated as MD):

MD property: If $y_{1}, y_{2} \in A, 0 \leqq y_{1}, 0 \leqq y_{2}, 0 \leqq u \leqq y_{1} y_{2}$, then there exists $u_{i} \in A, 0 \leqq u_{i} \leqq y_{i}(i=1,2)$ such that $u=u_{1} u_{2}$.

It was shown as Theorem 4 in [4] that if $A$ is commutative and has the MD property, then $A=A_{1}$. In this paper we will drop the commutativity assumption and show the following theorem:

MAIN THEOREM. If a dsc-pola $A$ has the MD property, then for each $x \in A, x=d+v$, where $d \in A_{1}, v^{2}=0$, and this expression is unique (in the sense that if $x=d+v=e+u, e \in A_{1}, u^{2}=0$, then $d=e, v=u$ ).

Received by the editors June 18, 1973.

AMS (MOS) subject classifications (1970). Primary 06A70; Secondary 15A45.

Key words and phrases. Dedekind $\sigma$-complete partially ordered linear algebra, nilpotent, multiplication decomposition property, matrix inequalities.

(c) American Mathematical Society 1974 
Lemma 1. For any dsc-pola $B$ if $w \in B, w^{2}=0$ and $w \geqq-1$, then $w \geqq 0$.

Proof. Since $1+w \geqq 0$, we have $(1+w)^{n} \geqq 0$ or $1+n w \geqq 0$, for all $n>0$. This means $w \geqq-(1 / n) 1$ for all $n$. By the Archimedean property we have $w \geqq 0$.

Lemma 2. For any dsc-pola $B$ if $w \geqq 0, w^{2}=0$, then for any $0 \leqq a \in B_{1}$ $\left(B_{1}\right.$ is the diagonal part of $\left.B\right),(a w)^{2}=(w a)^{2}=0$.

Proof. See the remark of Theorem II. 3.6 of [2].

LEMMA 3. If $a$ dsc-pola $B$ has the following property: given any $1 \leqq$ $x \in B, x^{-1}$ exists and $x^{-1} \leqq 1$, then for any $0 \leqq w \in B, w^{n}=0, n \geqq 2$, we have $w^{2}=0$; moreover, the sum (product) of positive nilpotents is a nilpotent (zero).

Proof. See Theorems II. 3.1, II. 3.2 and its corollary in [2].

Lemma 4. For any dsc-pola $B$, let $x \in B, 0 \leqq x \leqq 1$, if there exists $0 \leqq y$ such that $1 \leqq x y+y x$, then $x^{-1}$ exists and $x^{-1} \geqq 1$.

Proof. Put $z=1-x \geqq 0$. By assumption we have $1 \leqq x y+y x=$ $(1-z) y+y(1-z)$ or $1 \leqq 1+z y+y z \leqq 2 y$. Hence, $2 y \geqq 1+z\left(\frac{1}{2}\right)+\left(\frac{1}{2}\right) z=1+z$. By induction we will show $2 y \geqq 1+\sum_{k=1}^{n} z^{k}=h_{n}$ for all $n$. The assertion is clearly true for $n=1$. If the assertion is true for $n=m$, i.e., $2 y \geqq h_{m}$, then for $n=m+1$, we first observe that $2 y z \geqq h_{m} z, 2 z y \geqq z h_{m}$ and $h_{m} z=z h_{m}$; hence,

$$
2 y \geqq 1+y z+z y \geqq 1+\frac{1}{2}\left(h_{m} z+z h_{m}\right)=1+z h_{m}=1+\sum_{k=1}^{m+1} z^{k}=h_{m+1} .
$$

Therefore, $h_{n}$ is bounded above by $2 y$, by Proposition 2 in [3] we see

$$
1 \leqq h=\sup \left\{h_{n}\right\}=\sum_{0}^{\infty} z^{k}=(1-z)^{-1} \leqq 2 y .
$$

Theorem 5. Let the dsc-pola $A$ have the $M D$ property. If $0 \leqq x \in A$, then $x=c+w$, where $0 \leqq c \in A_{1}, 0 \leqq w$ and $w^{2}=0$.

Proof. Put $y=x+2 \geqq 2$. Clearly $1 \leqq y^{2}-1 \leqq y^{2}$. By the MD property there exists $z_{1}, z_{2} \in A$ such that $0 \leqq z_{1} \leqq y, 0 \leqq z_{2} \leqq y$ and $y^{2}-1=z_{1} z_{2}$. Thus

$$
1=y\left(y-z_{2}\right)+\left(y-z_{1}\right) z_{2}=\left(y-z_{1}\right) y+z_{1}\left(y-z_{2}\right) \text {. }
$$

From this we see easily that

$$
1 \geqq y\left(y-z_{2}\right) \geqq y-z_{2} \geqq 0, \quad 1 \geqq\left(y-z_{1}\right) y \geqq y-z_{1} \geqq 0 .
$$

Hence, $z_{1} \geqq y-1 \geqq 1, z_{2} \geqq y-1 \geqq 1$. Put $a=y-z_{1}, b=y-z_{2}$. Then $1 \geqq a y \geqq$ $a \geqq 0,1 \geqq y b \geqq b \geqq 0$; this means $a, b, a y, y b$ all belong to $A_{1}$; therefore, they 
commute with each other. Now $0 \leqq a+b \leqq 1=a z_{2}+y b \leqq a y+y b \leqq 2$. Thus,

$$
1 \leqq(a+b) y+y(a+b) \text {. }
$$

By Lemma 4 this implies $(a+b)^{-1}$ exists and $0 \leqq(a+b)^{-1} \in A_{1}$. Next observe that

and

$$
a(y a-a y)=(a y) a-a^{2} y=a(a y)-a^{2} y=0
$$

$$
\begin{aligned}
(y a-a y) b & =y(a b)-a y b=y(b a)-a y b=(y b) a-a y b \\
& =a(y b)-a y b=0 .
\end{aligned}
$$

Put $v=(y a-a y) a$. Then

and

$$
v^{2}=(y a-a y)(a(y a-a y)) a=0,
$$

$$
(y a-a y)(a+b)=(y a-a y) a+(y a-a y) b=v+0=v .
$$

Since $(a+b)^{-1}$ exists, we have $y a-a y=v(a+b)^{-1}$ or $y a=a y+v(a+b)^{-1}$.

Now note, by $1 \geqq y b, a y \geqq 0$, we have

$$
0 \leqq y(a+b)=y a+y b=y b+a y+v(a+b)^{-1} \leqq 2+v(a+b)^{-1} \text {. }
$$

Thus, $-2 \leqq-2(a+b) \leqq v$ (since $1 \geqq a+b \geqq 0$ ).

By Lemma 1 we have $v \geqq 0$. But from $0 \leqq y(a+b)=y b+a y+v(a+b)^{-1}$, and $(a+b)^{-1} \geqq 0$, we get

$$
y=(a y+y b)(a+b)^{-1}+v(a+b)^{-2}=c_{1}+w,
$$

where $0 \leqq c_{1}=(a y+y b)(a+b)^{-1} \in A_{1}, 0 \leqq w=v(a+b)^{-2}$.

By Lemma $2, w^{2}=0$. Finally, observe that $2(a+b) \leqq a y+y b$. Since $(a+b)^{-1} \geqq 0$, we obtain

$$
2 \leqq c_{1}=(a y+y b)(a+b)^{-1} \in A_{1} .
$$

Now $y=x+2=c_{1}+w$ or $x=c+w$, where $c=c_{1}-2 \geqq 0$. The proof is complete.

Corollary 6. If the dsc-pola $A$ has the MD property and if $u=u_{1} u_{2}=$ $u_{2} u_{1}$, where $u_{1}, u_{2}, u$ are as in the definition of the decomposition property, then $A=A_{1}$.

Proof. For any $1 \leqq x \in A$, we want to show $x^{-1} \geqq 0$. Choose $y \in A$, such that $1 \leqq x \leqq x+1 \leqq y$. Clearly $2 \leqq y$ and $1 \leqq y^{2}-1 \leqq y^{2}$. Thus, by the MD property and the assumption, there exists $0 \leqq z_{1} \leqq y, 0 \leqq z_{2} \leqq y$ such that $y^{2}-1=z_{1} z_{2}=z_{2} z_{1}$ or

$$
\begin{aligned}
1 & =y\left(y-z_{2}\right)+\left(y-z_{1}\right) z_{2}=\left(y-z_{1}\right) y+z_{1}\left(y-z_{2}\right) \\
& =y\left(y-z_{1}\right)+\left(y-z_{2}\right) z_{1} .
\end{aligned}
$$


Put $0 \leqq a=y-z_{1}, 0 \leqq b=y-z_{2}$. Then proceed as in Theorem 5. Note now $1 \geqq a y \geqq a \geqq 0,1 \geqq y a \geqq a \geqq 0$, so $a y, y a \in A_{1}$, hence, $y a-a y \in A_{1}$. This implies $v=(y a-a y) a \in A_{1}\left(v\right.$ as in the proof of Theorem 5). But $v^{2}=0$; this by Corollary I. 2.5 of [2] implies $v=0$. Therefore, $w=v(a+b)^{-2}=0$; hence, $2 \leqq y=c_{1}+w=c_{1} \in A_{1}$. This means $y^{-1} \geqq 0$. By Proposition 3 of [3] we see $x^{-1} \geqq 0$, hence, $x \in A_{1}$, thus, $A=A_{1}$.

COROLlaRY 7. If $A$ has the $M D$ property, then for any $1 \leqq x \in A, x^{-1}$ exists and $x^{-1} \leqq 1$.

Proof. From Theorem 5 we see easily that if $1 \leqq x \in A$, then $x=c+w$, where $1 \leqq c \in A_{1}, 0 \leqq w, w^{2}=0$. Since $0 \leqq c^{-1} \leqq 1$, we have $0 \leqq c^{-1} w \leqq w$, so $\left(c^{-1} w\right)^{2}=0$. Now $x=c\left(1+c^{-1} w\right)$, thus,

$$
x^{-1}=\left(1-c^{-1} w\right) c^{-1}=c^{-1}-c^{-1} w c^{-1} \leqq c^{-1} \leqq 1 .
$$

Remark. The converse of the theorem in general is not true; see the example at the end.

COROLlary 8. If $A$ has the $M D$ property, and $w \in A, w^{2}=0$, then $w=w_{1}-w_{2}$, where $0 \leqq w_{i} \in A, w_{i}^{2}=0(i=1,2)$, and $-v \leqq w \leqq v$ for some $0 \leqq$ $v \in A, v^{2}=0$.

Proof. Let $w=x_{1}-x_{2}, 0 \leqq x_{i}, i=1,2$. By Theorem $5 x_{i}=c_{i}+w_{i}$, where $0 \leqq c_{i} \in A_{1}, 0 \leqq w_{i}, w_{i}^{2}=0$, so $w=\left(c_{1}-c_{2}\right)+\left(w_{1}-w_{2}\right)$. Squaring both sides and using Corollary 7 and Lemma 3 we have

or

$$
w^{2}=0=\left(c_{1}-c_{2}\right)^{2}+\left(c_{1}-c_{2}\right)\left(w_{1}-w_{2}\right)+\left(w_{1}-w_{2}\right)\left(c_{1}-c_{2}\right)
$$

$$
-\left(c_{1}-c_{2}\right)^{2}=\left(c_{1}-c_{2}\right)\left(w_{1}-w_{2}\right)+\left(w_{1}-w_{2}\right)\left(c_{1}-c_{2}\right) .
$$

Squaring both sides again and repeatedly using Lemma 2, Lemma 3, and Corollary 7 , we have $\left(c_{1}-c_{2}\right)^{4}=0$. But $c_{1}-c_{2} \in A_{1}$; by Corollary I. 2.5 of [2] we see easily that $c_{1}-c_{2}=0$, so that $w=w_{1}-w_{2}$. By putting $v=w_{1}+$ $w_{2}$, and using Lemma 3 , the assertion is now clear.

REMARK. By the same method above, we can actually show that for any $w \in A$, if $w^{n}=0, n>2$, then $w^{2}=0$. Furthermore, by this corollary and Lemma 3, it is quite easy to see that the sum (product) of any two nilpotents is a nilpotent (zero).

Now the proof of the Main Theorem is straightforward as follows: For any $x \in A, x=x_{1}-x_{2}=\left(c_{1}+w_{1}\right)-\left(c_{2}+w_{2}\right)=d+w$, where $0 \leqq x_{i}=$ $c_{i}+w_{i}, 0 \leqq c_{i} \in A_{1}, 0 \leqq w_{i}, w_{i}^{2}=0(i=1,2)$, and $d=c_{1}-c_{2} \in A_{1}, w=w_{1}-w_{2}$. Note that $w^{2}=0$. For the uniqueness part: Suppose $x=d+w=e+u$, $e \in A_{1} u^{2}=0$. Then $d-e=u-w$. Squaring both sides and using the remark of Corollary 8 and Corollary I. 2.5 of [2], we see immediately that $d=e$ and $u=w$. 
Let $N=\left\{w: w \in A, w^{2}=0\right\}$. From Corollary 8 and its remark we know $N$ is an additive group; it is trivial to verify that $N$ is a dsc-pola. Now we show that $N$ has the well-known addition decomposition property:

THEOREM 9. If $u_{i} \in N, u_{i} \geqq 0(i=1,2)$ and $0 \leqq w \leqq u_{1}+u_{2}$, then there exists $0 \leqq w_{i} \leqq u_{i}$ such that $w=w_{1}+w_{2}$.

Proof. Since $0 \leqq w \leqq\left(1+u_{1}\right)\left(1+u_{2}\right)=1+u_{1}+u_{2}$, by the MD property, we have $w=z_{1} z_{2}$ where $0 \leqq z_{i} \leqq 1+u_{i}(i=1,2)$. By Theorem 5 we obtain easily that $z_{i}=a_{i}+v_{i}$, where $0 \leqq a_{i} \leqq 1,0 \leqq v_{i} \leqq u_{i}$. Now

$$
w=\left(a_{1}+v_{1}\right)\left(a_{2}+v_{2}\right)=a_{1} a_{2}+a_{1} v_{2}+v_{1} a_{2} .
$$

This implies $0 \leqq a_{1} a_{2} \leqq w$. Therefore, $\left(a_{1} a_{2}\right)^{2}=0$. But $a_{1} a_{2} \in A_{1}$, hence, $a_{1} a_{2}=0$. Now by putting $w_{1}=v_{1} a_{2}, w_{2}=a_{1} v_{2}$, then the assertion is clear.

EXAMPLE 1. Let $A$ be the real linear algebra of matrices (real entries) of some given finite order. If $A$ is partially ordered componentwise, then the diagonal part $A_{1}$ of $A$ is nothing but all the diagonal matrices. If, in particular, $A$ consists of the matrices which have the form $x=\left[\alpha_{i j}\right]$ where $\alpha_{i j}=0$ for $i \neq j$ or $i \neq 1$, then the readers are invited to verify that $A$ has the MD property. Note each element in $A$ can be written as a diagonal matrix plus a nilpotent matrix.

EXAMPLE 2. $A\left\{\left[\begin{array}{ll}\alpha & \delta \\ 0\end{array}\right]: \alpha, \delta\right.$ are reals $\}$. If we order $A$ componentwise, then $A$ is a dsc-pola. It can be verified easily that $A$ has no MD property, but each element of $A$ can be decomposed as a diagonal matrix plus a nilpotent matrix. This means the converse of the Main Theorem is not true.

ACKNOWLEDGEMENT. This problem was brought to the author's attention by Professor R. DeMarr. The author wishes to thank him for his valuable criticism in preparing this work.

\section{REFERENCES}

1. G. Birkhoff, Lattice theory, 3rd. ed., Amer. Math. Soc. Colloq. Publ., vol. 25, Amer. Math. Soc., Providence, R.I., 1967. MR 37 \#2638.

2. T. Y. Dai, On some specially classes of partially ordered linear algebras, J. Math. Anal. Appl. 40 (1972), 649-682.

3. R. E. DeMarr, On partially ordering operator algebras, Canad. J. Math. 19 (1967), 636-643. MR 35 \#3450.

4. —_ A class of partially ordered linear algebras, Proc. Amer. Math. Soc. 39 (1973), 255-260.

Department of Mathematics, York College, City University of New York JAMAICA, NeW YORK 11432 